\title{
Ischemic Succinate Accumulation Inhibits Neural Stem Cell Proliferation after Cerebral Ischemia/Reperfusion via Inducing Cdc42 Succinylation
}

\section{Linyan Huang}

Xuzhou Medical University

Juyun Ma

Xuzhou Medical University

Jinxiu Song

Xuzhou Medical University

Jingjing Xu

Xuzhou Medical University

Haidi Fan

Xuzhou Medical University

Heng Cai

Xuzhou Medical University

Wan Wang

Xuzhou Medical University

Yanling Wang

Xuzhou Medical University

Zhaoli Hu

Xuzhou Medical University

Jiangang Shen

The University of Hong Kong

Suhua Qi ( $\sim$ suhuaqi@xzhmu.edu.cn )

Xuzhou Medical University

\section{Research}

Keywords: Succinate, Neural stem cell, Cdc42, Succinylation, Cerebral ischemia/ Reperfusion Injury Posted Date: September 8th, 2021

DOI: https://doi.org/10.21203/rs.3.rs-871703/v1 
License: (c) (i) This work is licensed under a Creative Commons Attribution 4.0 International License. Read Full License 


\section{Abstract}

\section{Background}

Neural stem cell proliferation is a critical process in endogenous neurogenesis after ischemic/reperfusion injury. Ischemic succinate accumulation causes cerebral damage due to excess reactive oxygen species production. However, it remains elusive whether ischemic succinate accumulation affects neural stem cell proliferation.

\section{Methods}

In this study, we established rat middle cerebral artery occlusion model to mimic ischemic/reperfusion injury in vivo. Primary neural stem cell and neural stem cell line C17.2 were challenged with oxygenglucose deprivation and reoxygenation. The level of succinate was assessed by liquid chromatographymass spectrometry analysis. Cell proliferation was tested by cell counting kit 8 . The infarct volume was measured by 2,3,5-Triphenyltetrazolium chloride staining. G protein coupled receptor GPR91 was detected by immunofluorescence. Sirtuin 5 knockdown was performed with Sirt 5 siRNA transfection. Cdc42 GTPase activity and succinylation were evaluated by co-immunoprecipitation.

Results

We showed that succinate increased in the serum and brain (cortex and hippocampus) in middle cerebral artery occlusion rats. oxygen-glucose deprivation also induced abundant succinate in neural stem cell. Diethyl succinate inhibited C17.2 cell proliferation. Intraperitoneal administration of DS $(800 \mathrm{mg} / \mathrm{kg})$ increased the infarct volume in middle cerebral artery occlusion rats. Besides, Diethyl succinate increases Cdc42 succinylation but represses Rho family GTPase Cdc42 activity, without affecting the level of its receptor $\mathrm{G}$ protein-coupled receptor-91 in neural stem cells. Increasing Cdc42 succinylation by desuccinylacylase Sirt5 knockdown inhibited GTPase Cdc42 activity. Accordingly, Cdc42 inhibtor ML141 also inhibited C17.2 proliferation.

\section{Conclusions}

Our observations showed that accumulation of succinate inhibited neural stem cell proliferation by inducing Cdc42 succinylation, which reduced Cdc42 GTPase activity and was detrimental for neurogenesis after ischemic/reperfusion injury. Our data revealed a new mechanism that ischemic succinate accumulation aggrevated cerebral ischemic/reperfusion injury.

\section{Introduction}

Cerebral ischemic/reperfusion occur when cerebral blood supply is disrupted and then restored. The reperfusion initiates oxidative damage and cell death through generation of mitochondrial reactive oxygen species (ROS)(Eltzschig and Eckle, 2011). The metabolomics analysis and several reports showed that mitochondrial ROS production was driven by ischemic succinate(an intermediate of citric 
acid cycle) accumulation(Chouchani et al., 2014; Kamarauskaite et al., 2020; Zhang et al., 2020). A potentially therapeutically relevant effect of ischemic/reperfusion injury is the robust stimulation of neurogenesis in the adult brain(Gu et al., 2000). De novo neurogenesis has been found in the subventricular zone (SVZ) of the adult mammalian brain after stroke. Cerebral ischemic/reperfusion injury induces cellular proliferation in the SVZ, migration from the SVZ to the peri-infarct tissue of immature and still proliferating neurons (neuroblasts), and differentiation into neurons(Donega and Raineteau, 2017). Under aerobic condition, succinate is rapidly metabolized into fumarate, however, ischemic succinate accumulation arises from reversal of succinate dehydrogenase. So far, aberrant succinate accumulation has been reported to link with cardiomyocyte hypertrophy(Aguiar et al., 2014), myocardial infarction(Kohlhauer et al., 2018), inflammation(Lampropoulou et al., 2016), skeletal muscle fiber remodeling(Wang et al., 2019), osteoclastogenesis(Guo et al., 2017). Decreasing ischemic succinate accumulation by pharmacological inhibition is sufficient to ameliorate in vivo IR injury in murine models of cardiovascular and renal disease(Jiang et al., 2019; Beach et al., 2020). However, it remains unclear how succinate accumulation of impact neurogenesis after ischemic/reperfusion injury.

The role of succinate includes metabolic and non-metabolic pathway. It has been reported that succinate binds to and activates G-protein coupled receptor, GPR91, and triggers intracellular $\mathrm{Ca}^{2+}$ and cAMP, as well as mitogen-activated protein kinases. Succinate induced GPR91 activation contributes to the ischemic injury in several kinds of diseases, like cardiomyocyte hypertrophy and muscle remodeling(Aguiar et al., 2014; Wang et al., 2019). Except that, succinylation is a newly discovered and multienzyme-regulated post-translational modification (PTM). The form of succinylation is composed of non-enzymatic succinylation by succinyl CoA and enzymatic succinylation(Yang and Gibson, 2019). Succinate and succinyl CoA can be converted into each other under the action of succinyl-CoA synthetase and succinyl-CoA ligase, thus regulating succinylation. Improving succinate production by succinate dehydrogenase inactivation induces hypersuccinylation by causing the accumulation of succinyl CoA(Zhang et al., 2011; Li et al., 2015). Hypoxia increase succinylation(Gibson et al., 2015; Chen et al., 2017) and succinylation may alter the response to heart and brain injury. Hypersuccinylation leads to hypertrophic cardiomyopathy(Sadhukhan et al., 2016). The regulation of succinylation also links to brain ischemic tolerance(Koronowski et al., 2018), for example, Sirtuin protein 5 (desuccinylase) knockout mice are more susceptible to ischemia-reperfusion injury compared with wildtype littermates. The succinylation of specific protein in metabolism pathway alters its activity, like isocitrate dehydrogenase. Hence, it deserves further investigation how succinylation modulates neurogensis after ischemic/reperfusion injury.

Here we aim to observe the level of succinate after cerebral ischemia/reperfusion injury, and the effect of the altered metabolite, succinate, on the process of neurogenesis, like NSC proliferation. Next, we try to figure out the mechanism how succinate regulates NSC proliferation to affect ischemic stroke outcome. Our data provide a new point to explain the functional role of ischemic succinate accumulation in ischemic/reperfusion injury. 


\section{Materials And Methods}

\section{Animal model of ischemia/reperfusion injury}

All animals were kept under a constant temperature $\left(23 \pm 2^{\circ} \mathrm{C}\right)$ and a light-dark cycle of 12 hours $(\mathrm{h})$. The animals were fed with commercially available normal rat diet and tap water ad libitum. Adult male Sprague Dawley rats (Shanghai Experimental Animal Center, Chinese Academy of Science), weighing 250 to $300 \mathrm{~g}$, were used in this study. The middle cerebral artery occlusion model was induced following an established method described previously(Hu et al., 2020). Briefly, rats were anesthetized with $1.5 \%$ Phenobarbital sodium. Then the common, external and internal carotid arteries were exposed through a ventral midline incision. A silicon coated 4-0 monofilament nylon suture with rounded tip was inserted through the right common carotid artery, and advanced until it occluded the middle cerebral artery. Then the anesthesia was reversed. After $1 \mathrm{~h}$ of occlusion, the rat was re-anesthetized and the nylon suture was withdrawn. Rectal temperature was maintained around $37^{\circ} \mathrm{C}$ throughout the surgical procedure using a feedback-regulated heating system.

The rats were randomly divided into MCAO, MCAO + Diethyl succinate (Sigma-Aldrich, labeled as DS). DS was injected intraperitoneally before ischemia for $0.5 \mathrm{~h}$, followed with ischemia for $1 \mathrm{~h}$ and reperfusion for $23 \mathrm{~h}$. At least six rats were included in each group. TTC staining was evaluated by an independent pathologist.

\section{Cell culture}

\section{Primary neural stem cell culture}

The pregnant rats (E14-E19d) were anesthetized with ether and fixed on the plate after decapitation. The rats were sprayed with $75 \%$ alcohol and disinfected. Take out the fetus, rinse once with ultrapure water containing $1 \%$ penicillin/streptomycin(P/S) and put them in the peri dish filled with pre-cold Dulbecco's modified Eagle's medium (DMEM)-F12 containing 1\%P/S. Dissect the fetus brain, and the hippocampus and cortex were separated under the microscope. Chop the hippocampus and cortex, pour into a $50 \mathrm{ml}$ centrifuge tube, centrifuge for $10 \mathrm{~s}$. Add $4 \mathrm{ml}$ trypsin into the pellet; digest in a $37^{\circ}$ water bath for $15-25 \mathrm{~min}$ with gently shaking. After filtration, add $50 \mathrm{ml}$ stop solution (DMEM-F12 containing $1 \% \mathrm{P} / \mathrm{S}$ ), and spin down. Add NSC basal medium (DMEM-F12 100ml, 2\% B27 2ml, EGF 10ug/ul 100ul, FGF 10ug/ul 100ul, $0.5 \% \mathrm{P} / \mathrm{S}$ and $1 \mathrm{mM}$ Gln $500 \mathrm{ul}$ ) to the cell pellet, resuspend and inoculate into the culture flask. After 24 hours, aspirate the medium into a new tube, centrifuge at $600 \mathrm{r} / \mathrm{min}$ for 5 minutes, discard half of the supernatant, add half of the new medium and resuspend. Add 1-2 $\mathrm{ml}$ of medium every other day. On the 11 th day, the neurospheres can be seen under the microscope. The neurospheres were centrifuged at $1000 \mathrm{r} / \mathrm{min}$ for $5 \mathrm{~min}$. The supernatant was discarded, and the cells were washed with PBS on ice. Digest with $2 \mathrm{ml}$ diluted trypsin, shake in a $37^{\circ} \mathrm{C}$ water bath for 15 minutes, and add $10 \mathrm{ml}$ of DMEM-F12 medium to terminate digestion. Then spin down, add $10 \mathrm{ml}$ of complete NSC medium (NSC basal medium with $20 \%$ fetal bovine serum (FBS)), and filter through a 200 -mesh nylon to obtain the neural stem cell suspension. 


\section{Neural stem cell line $\mathbf{C} 17.2$}

The multipotent NSC line C17.2 was gifted from Professor Jiangang Shen (School of Chinese Medicine, Li Ka Shing Faculty of Medicine, The University of Hong Kong, 10 Sassoon Road, Pokfulam, Hong Kong, SAR, China). C17.2 cells were cultured in Dulbecco's modified Eagle's medium (DMEM; Invitrogen) containing $10 \% \mathrm{FBS}$ and $2 \mathrm{mM} \mathrm{L-glutamine}$ at $37^{\circ} \mathrm{C}$ in humidified incubators with $5 \% \mathrm{CO}_{2}$.

\section{Oxygen and Glucose Deprivation Reoxygenation}

To mimic the ischemic stroke condition in vitro, primary neural stem cells and C17.2 cells were subjected to oxygen and glucose deprivation (OGD) treatment. In brief, cell culture medium was replaced with DMEM medium without glucose. Cells were incubated in a humidified airtight chamber in which the atmosphere was saturated with $95 \% \mathrm{~N}_{2} / 5 \% \mathrm{CO}_{2}$ at $37^{\circ} \mathrm{C}$. The oxygen concentration was less than $1 \%$ as monitored by an oxygen analyzer (Sable Systems, Las Vegas, NV, USA). For the control group, cells were replaced with fresh DMEM medium and incubated at $37^{\circ} \mathrm{C}$ in a humidified incubator with $5 \% \mathrm{CO}_{2}$ and $95 \%$ room air.

\section{Measurement of succinate by liquid chromatography-mass spectrometry analysis}

The serum and cerebral spinal fluid were collected from normal control and MCAO rats, then the rats were sacrificed, the ischemic cortex and hippocampus, as well as the cecum contents were collected for homogenizing. After centrifuge at $3000 \mathrm{r} / \mathrm{min}$ for $10 \mathrm{~min}$, the supernatant was aspirated for succinate assay. Before detection, $50 \mu \mathrm{L}$ samples were mixed with $195 \mu \mathrm{L}$ methanol containing $10 \mu \mathrm{mol} / \mathrm{L}$ 2,2,3,3tetradeuteriobutanedioic acid (as internal standard, IS) for $2 \mathrm{~min}$, then spinned down for $10 \mathrm{~min}$ at 14000 rpm, and collected $150 \mu \mathrm{L}$ for LC-MS/MS.

All samples were analyzed in multiple reactions monitoring (MRM) mode using the UPLC/TQD system (Waters, Milford, MA) equipped with an electrospray ionization (ESI) probe as the interface. Analyses were performed on an Acquity UPLC BEH C18 column $(2.1 \times 50 \mathrm{~mm} ; 1.7 \mathrm{~mm}$; Waters, Ireland). The mobile phase was maintained at a flow rate of $0.4 \mathrm{~mL} / \mathrm{min}$, containing $0.1 \%(\mathrm{v} / \mathrm{v})$ formic acid water solution $(\mathrm{A})$ and $0.1 \%(\mathrm{v} / \mathrm{v})$ formic acid acetonitrile solution (B). Succinic acid was separated by gradient of: $10 \%$ buffer $B$ for $0.2 \mathrm{~min}, 10 \%$ buffer $B$ to $80 \%$ buffer B for $1.8 \mathrm{~min}, 80 \%$ buffer $B$ for $0.5 \mathrm{~min}, 80 \%$ buffer $B$ to $10 \%$ for $0.5 \mathrm{~min}$, holding with $10 \%$ buffer $B$ for $1.5 \mathrm{~min}$. The temperature of the column was maintained at $40 \mathrm{oC}$. The ESI source was set in negative ionization mode. Quantification was performed using multiple reaction monitoring (MRM) with $\mathrm{m} / \mathrm{z} 117 \rightarrow 73$ for succinic acid quantification, $117 \rightarrow 98$ for succinic acid identification, and $121 \rightarrow 77$ for IS quantification, $121 \rightarrow 102$ for IS identification. The optimal MS parameters were: capillary voltage of $1.16 \mathrm{kV}$, cone voltage of $24 \mathrm{~V}$, collision voltage of $12 \mathrm{~V}$, source temperature of $150 \mathrm{oC}$ and desolvation temperature of $600 \mathrm{oC}$. The desolvation flow rate was $600 \mathrm{~L} / \mathrm{h}$.

\section{2,3,5-Triphenyltetrazolium chloride (TTC) staining}


TTC staining is commonly applied for the visualization of hypoxic brain tissue and for defining the size of cerebral infarction and penumbra(Chen et al., 2019). After $1 \mathrm{~h}$ of ischemia and reperfusion for $23 \mathrm{~h}$, the animals were sacrificed and the brains were quickly dissected. The brains were placed in a refrigerator at $-20^{\circ} \mathrm{C}$ for $30 \mathrm{~min}$. Then the brain was cut into $2 \mathrm{~mm}$ slice, stained with 2\% TTC (A610558, Shanghai Sangon), and incubated in a $37^{\circ} \mathrm{C}$ oven for $25 \mathrm{~min}$. The brain slices were photographed and preserved in $4 \%$ paraformaldehyde solution. Infarct percentage $=$ (uninfarct hemisphere area-infarct hemisphere uninfarct area)/uninfarct hemisphere area $\times 100 \%$.

\section{Cell viability assay}

C17.2 cells were plated in 96-well cell culture plates at the concentration of $5 \times 10^{3}$ cells/well. After $24 \mathrm{~h}$, fresh medium with DS at different concentrations $(0.5,1,2,5,10 \mathrm{mM})$ was added. After $48 \mathrm{~h}$, cell proliferation was determined by Cell Counting Kit 8 (CCK8) kit (Dojindo, Tokyo, Japan) following the manufacturer's instructions.

\section{Immunofluorescence staining}

For immunofluorescence staining, paraffin sections were dewaxed and treated by antigen retrieval. Then the sections were treated with phosphate-buffered saline containing $0.1 \%$ Triton X-100, $5 \%$ donkey serum for $1 \mathrm{~h}$. After incubation with primary antibodies against GPR91 and Nestin at $4^{\circ} \mathrm{C}$ overnight, the samples were incubated for 30 min with appropriate FITC- or Cy3-labelled secondary antibodies (Jackson ImmunoResearch Laboratory) at room temperature for $1 \mathrm{~h}$. The nuclei were stained with DAPI. Slides were then examined with an Olympus microscope, and images were analyzed with software.

\section{Immunoblotting}

Western blot was performed as previously described(Huang et al., 2014). Briefly, C17.2 cells were lysed with RIPA lysis buffer containing protease inhibitor cocktail (Merk, Germany). Protein was separated with $10 \%$ SDS-PAGE and was transformed to polyvinylidene fluoride membranes (Millipore, USA). The membranes were blocked in 5\% non-fat dry milk diluted with TBST (in mM: Tris- $\mathrm{HCl} 20, \mathrm{NaCl} 150, \mathrm{pH} 7.5$, $0.1 \%$ Tween 20 ) at room temperature for $1 \mathrm{~h}$. The membranes were incubated with primary antibody: antiGPR91 (1:1000 dilution; Cell Signaling Technology, USA), anti-Sirt5 (1:1000 dilution, Cell Signaling Technology, USA), anti-Cdc42 (1:1000 dilution; Cell Signaling Technology, USA), Pan anti-succinylysine (1:1000 dilution; PTM Biolabs, China), at $4^{\circ} \mathrm{C}$ overnight, and then were incubated for $1 \mathrm{~h}$ with anti-rabbit IgG conjugated to horseradish peroxidase (1:1000 dilution; Cell Signaling Technology, USA) at room temperature. Polyclonal rabbit $\beta$-Actin antibody (1:1000 dilution; Santa Cruz Biotechnology Inc., USA) or a-tubulin antibody (1:1000 dilution; Cell Signaling Technology, USA) was performed as the loading control. All bands were detected with Pierce ECL western blotting substrate (Bio-rad, USA).

\section{Measurement of Cdc42 GTPase activity}

Cdc42 GTPase activity was performed using the Rac1/Cdc42 activation assay kit (Sigma-Aldrich, Cat. No. 17-441). Briefly, cells were lysed with $300 \mu \mathrm{L}$ modified lysis buffer. Add Rac/cdc42 Assay Reagent directly to the lysate immediately after removing cellular debris and insoluble material by centrifugation. 
Gently rock the reaction mixture at $4^{\circ} \mathrm{C}$ for 60 minutes. Collect the agarose beads by pulsing ( 5 seconds in the microcentrifuge at $14000 \mathrm{~g}$ ) and drain off the supernatant. Wash the beads 3 times with MLB. Resuspend the agarose beads in an appropriate amount of $2 \times$ Laemmli sample buffer and boil for 5 minutes. Use $20 \mu \mathrm{l}$ per assay for $10 \mathrm{~cm} \times 10 \mathrm{~cm}$ mini gels. The supernatant was collected for SDS-PAGE and subsequent immunoblot analysis. Probe the blot with anti-Cdc42, followed by HRP-conjugated secondary antibody and ECL reagent.

\section{Co-immunoprecipitation (IP) of Cdc42 Succinylation}

Use $200 \mu \mathrm{L}$ IP lysis buffer to lyse cells, assaying protein concentration after removing cellular debris and insoluble material by centrifugation. Adjust protein concentration to $2 \mu \mathrm{g} / \mu \mathrm{L}$ using IP lysis buffer. Add Succinylated antibody and Cdc42 antibody respectively to the lysate and gently rock the reaction mixture at $4^{\circ} \mathrm{C}$ overnight. Wash the beads 3 times with PBST, then add the beads to the reaction mixture and gently rock the mixture at $4^{\circ} \mathrm{C}$ for $2.5 \mathrm{~h}$. Remove the supernatant on magnetic frame. Wash the beads 3 times with PBST. Resuspend the beads in an appropriate amount of $2 \times$ loading buffer and boil at $95^{\circ} \mathrm{C}$ for 5 minutes. Subsequent western blot analysis can be performed.

\section{Sirt5 siRNA transfection}

Add $125 \mu \mathrm{L}$ Opti-MEM in two eppendorff tubes, and then add $10 \mu \mathrm{L}$ lipo 3000 transfection solution and $10 \mu \mathrm{L}$ Sirt5 siRNA respectively. Set $5 \mathrm{~min}$ after oscillation, then mix and oscillate for $15 \mathrm{~min}$, add the mixture to the cell and replace with complete medium after $6 \mathrm{~h}$.

\section{Statistical analysis}

Statistical analysis was performed using the SPSS 19.0 software (SPSS, Inc, Chicago, IL). All experimental data were expressed as mean \pm SD. The comparison of succinate (in vivo), infarct volume, GPR91 and SIRT 5 expression was analyzed with unpaired $t$ test. The comparison of succinate (in vitro) was analyzed with repeated measurement ANOVA. The data for CCK8 were analyzed with one-way ANOVA, followed by Dunnet $t$ test. The comparison of Cdc42 level was analyzed with 1- or 2-way ANOVA, followed by Tukey test. The values were considered significant when $\mathrm{P}$ was $<0.05$.

\section{Results}

\section{Abnormal succinate accumulated under cerebral ischemic/reperfusion injury.}

In order to determine whether succinate is produced in large quantities in MCAO, we used LC-MS / MS technology to detect the level of succinate in different tissues from Sham and MCAO rats. The succinate concentration $(\mu \mathrm{M})$ was obviously higher in the serum $(1.55 \pm 0.23$ vs $2.11 \pm 0.08, P<0.05)$, cortex ( $0.81 \pm 0.17$ vs $2.68 \pm 0.55, P<0.01)$ and hippocampus $(0.34 \pm 0.16$ vs $1.77 \pm 0.27, P<0.01)$ in MCAO rats, while it was not comparable in cerebrospinal fluid $(0.59 \pm 0.06$ vs $0.64 \pm 0.11, P>0.05)$ and ceacum $(32.9493 \pm 4.05$ vs $38.579 \pm 8.36, P>0.05)$ (Fig 1A). To test whether succinate participates in the NSC proliferation after ischemic/reperfusion injury, we established a stroke model on primary neural stem cells. The results showed that the normal intracellular succinate content was $0.36 \pm 0.08 \mu \mathrm{M}$, and after $2 \mathrm{~h}$ 
of oxygen and glucose deprivation, succinate increased to $3.28 \pm 0.19 \mu \mathrm{M}(P<0.01$, vs Con $)$, which decreased to the basal level immediately after reoxygenation for $10 \mathrm{~min}$ and maintained this level until 24 h (Fig. 1B).

\section{Diethyl succinate inhibited NSC proliferation under physiological and OGD condition.}

Neurogenesis after stroke is a multiple process comprising several steps like, proliferation of neural stem/progenitor cells (NSPCs), differentiation of new neuroblasts to phenotype of neurons and survival of immature or mature neurons. Under physiological condition, Diethyl succinate (DS), a derivative of succinate, is used. DS (0.5-10 mM) inhibited neural stem cell line C17.2 proliferation in a dose-dependent manner $(0.99 \pm 0.11,0.88 \pm 0.08,0.76 \pm 0.07,0.62 \pm 0.09,0.45 \pm 0.06, v s 1 \pm 0.01, P>0.05, P<0.01, P<0.0001$, $P<0.0001, P<0.0001, \mathrm{n}=3$ ) (Fig. 2A). Under oxygen and glucose-deprivation, DS also inhibited C17.2 proliferation in a dose-dependent manner $(0.57 \pm 0.06,0.54 \pm 0.08,0.47 \pm 0.07,0.34 \pm 0.08,0.16 \pm 0.03$, vs $0.70 \pm 0.05, P>0.05, P<0.05, P<0.01, P<0.0001, P<0.0001, \mathrm{n}=3)$ (Fig. 2B).

\section{Administration of succinate aggravates ischemic/reperfusion injury severity.}

To observe the role of succinate in ischemic/reperfusion injury, we injected the rats of MCAO with DS $(800 \mathrm{mg} / \mathrm{kg})$ intraperitoneally. We found that DS injection caused the larger infarct volume in MCAO rats $(37.15 \pm 5.89 \%$ vs $25.51 \pm 6.72 \%, P<0.05, n=6)$ (Fig 4$)$. It demonstrated that succinate accumulation promotes ischemic/reperfusion injury.

\section{Succinate receptor GPR91 was independent on DS-inhibited C17.2 proliferation.}

The orphan G protein-coupled receptor GPR91 is responsible for the biological functions of succinate beyond energy production. So we speculated that DS might have influence on the function of GPR91, as GPR91 can bind G protein like Gai or Gq. Once Gai is activated, it will cause the inactivation of Rho family GTPase Cdc42. However, whether GPR91 exists in neural stem cells (NSCs) remain elusive. Hence, we examine the expression of GPR91 in primary NSCs (Nestin is used as the marker). The co-localization immunofluorescence assay showed that there are pronounced expression of GPR91 in primary NSCs (Fig. 4A). However, the intensity of GPR91 expression was unaffected after DS trigger (Fig. 4B,C). Compared with control group, the level of GPR91 was significantly increased in $\mathrm{C} 17.2$ cells challenged with OGD(Fig. 4D,E). To investigate whether GPR91 is involved in DS-inhibited cell proliferation, we assessed the effect of GPR91 knockdown using CCK8. We found that no matter in physiological or OGD condition, although DS inhibited cell proliferation, GPR91 knockdown could not reverse this effect $(0.81 \pm 0.08$ vs $0.80 \pm 0.09, P>0.05 ; 0.76 \pm 0.06$ vs $0.77 \pm 0.06, P>0.05 ; n=3)(F i g .4 F, G)$. This demonstrated that succinate receptor GPR91 is dispensable for DS-inhibited C17.2 proliferation.

\section{Succinate inhibits GTP-Cdc42 activity by increasing Cdc42 succinylation.}

As succinylation is a novel protein post-translational modification, we hypothesize that succinate might affect the downstream target of GPR91, like Cdc42 through succinylation. Therefore, we measured the Cdc42 GTPase activity and succinylation through co-immunoprecipitation, and we find that DS or OGD 
single or combined stimulation does not change the level of total Cdc42 (Fig. 5A,B), while DS treatment significantly inhibits GTP-Cdc42 activity(Fig. 5A,C) and Cdc42 succinylation (Fig.5D) under OGD. Sirt5 is the well-known desuccinylase, when we knockdown sirt5 in C17.2 cells, we found that Cdc42 succinylation rose while Cdc42 GTPase activity decreased (Fig.5E-G). This demonstrated that Cdc42 succinylation might weaken Cdc42 GTPase activity.

\section{ML141 inhibits C17.2 proliferation after OGD challenge.}

ML141 is a potent and selective inhibitor of Cdc42, to confirm if the mechanism of DS acting on C17.2 proliferation was through inhibiting Cdc42 activity, we compared the effect of DS and ML141 simultaneously. Compared with Con, OGD reduced C17.2 proliferation ratio $(0.78 \pm 0.08$ vs $1 \pm 0.11, P<0.01$, $\mathrm{n}=3)$. Compared with OGD, DS $(1,5,10 \mathrm{mM})$ inhibited cell proliferation ratio $(0.62 \pm 0.06,0.57 \pm 0.05$, $0.46 \pm 0.09$ vs $0.78 \pm 0.08, P<0.05, P<0.01, P<0.01, \mathrm{n}=3)$. Also, compared with OGD, ML141 $(1,5,10 \mathrm{mM})$ inhibited cell proliferation ratio $(0.59 \pm 0.07,0.55 \pm 0.12,0.14 \pm 0.01$ vs $0.78 \pm 0.08, P<0.01, P<0.01, P<0.0001$, $\mathrm{n}=3$ ). This indicated that under OGD condition, DS-inhibited C17.2 proliferation might be attributed to suppressing Cdc42 activity (Fig.6).

\section{Discussion}

Our study shows that abundant succinate appears in the serum, cortex and hippocampus of MCAO rats. Succinate shows the ability to inhibit NSC proliferation after cerebral ischemic/reperfusion injury, and the mechanism is mainly through repressing Cdc42 GTPase activity by increasing Cdc42 succinylation(Graphic abstract, Fig. 7). From the neural stem cell view, our data expands the mechanism of ischemic accumulation of succinate causing cerebral ischemic/reperfusion injury.

The existing data show that under steady state, circulating level of succinate varys from 2 to $20 \mu \mathrm{m}$, and increases exponentially under pathological conditions including ischemia-reperfusion injury following myocardial infarction(Kohlhauer et al., 2018). It was also reported that Ischemic accumulation of succinate caused reperfusion injury via mitochondrial ROS(Chouchani et al., 2014). Indeed, we found high levels of succinate, both in serum, cortex and hippocampus of the rat MCAO model (Fig. 1A). Hence, it is deserved to explore the role of these tissue inherent succinate on neurological function.

As NSCs also produced comparable amount of succinate after OGD (Fig. 1B), hence, we chose diethyl succinate, a membrane-permeable succinate, which is able to increase intracellular succinate(Tannahill et al., 2013; Li et al., 2017), to study the role of intracellular succinate on NSC. As expected, DS inhibited NSC proliferation under OGD condition (Fig. 2). Also, DS injection increases the infarct volume of MCAO rats (Fig. 3). This demonstrates that DS administration is detrimental to the MCAO severity. In other words, DS-inhibited proliferation plays the dominant role in neural damage after ischemic/reperfusion injury.

Increasing researches reveal that the function of succinate may be classified as metabolic or nonmetabolic which depends on the distribution of sccinate(Grimolizzi and Arranz, 2018). In mitochondria, 
succinate plays a crucial role in metabolism and operates in both anabolic and catabolic pathways(Tannahill et al., 2013; Kelly and O'Neill, 2015). In the cytosol, elevated cytosolic succinate levels may promote protein post-translational modifications by addition of succinyl groups to lysine residues(Xie et al., 2012; Park et al., 2013). Additionally, succinate may be released to the extracellular space through plasma membrane transporters of the SLC13 family(Willmes and Birkenfeld, 2013). Extracelluar succinate affects surrounding microenvironment by GPR91-mediated signal transduction mechanism(Rubic et al., 2008). Consistently, we also confirmed that the effect of DS (intracellular succinate) on NSC proliferation is not related to GPR91 (Fig. 4).

Many cellular processes are under the control of different small GTPases of the Rho family, including RhoA, Rac1 and Cdc42 (cell division cycle protein)(Nobes and Hall, 1995). These GTPases possess different functions, recent studies have also demonstrated that Cdc42 is implicated in a variety of biological activities in the nervous system, such as cell signaling, cytoskeleton organization, establishment of neuron polarity and regulation of cell morphology, motility and cell cycle progression(Etienne-Manneville and Hall, 2003). The effector proteins are able to recognize the activated form and induce conformational changes in Cdc42 (especially the switch I and switch II loops), which are critical for the subsequent signaling pathway. The relationship between Cdc42 activation and ischemia/reperfusion injury was also proved(Yang et al., 2019). Our results demonstrated that, after OGD exposure, DS did not alter total Cdc42 level, however, reduced Cdc42 GTPase activity in NSCs (Fig. 5A-C).

Cdc42 activity is also regulated by its various post-translational modifications (PTMs), like monophosphate adenylation, phosphorylation and glycosylation. One such PTM is lysine succinylation, which is regulated by sirtuin 5 (SIRT5)(Zhang et al., 2011; Park et al., 2013). Lysine succinylation is an important regulator of cellular processes, connecting to metabolism, inflammation and microbiota infection(Park et al., 2013; Ren et al., 2018; Yang and Gibson, 2019). Whether Cdc42 succinylation is also involved in the regulation of Cdc42 activity has been unclear yet. We found that DS treatment reduced Cdc42 activity, but the succinylation of Cdc42 increased (Fig. 5D). To confirm whether Cdc42 succinylation affects Cdc42 activity, we increased the Cdc42 succinylation by Sirt5 knockdown, and found that Cdc42 activity was repressed (Fig. 5E-G). Furtherly, as supposed, decreased Cdc42 activity using Cdc42 inhibitor ML141 also inhibited NSC proliferation like DS (Fig. 6).

\section{Conclusions}

Our research expands the mechanism of succinate ischemic accumulation causing reperfusion injury. Unexpectedly, it damages NSC proliferation through succinylating Cdc42 which is harmful for neurorestorative after ischemic/reperfusion injury. Our data support that modulation of succinate level or Cdc42 activity might be a potential therapeutic target for ischemic/reperfusion injury.

\section{Abbreviations}

MCAO middle cerebral artery occlusion 
OGD oxygen-glucose deprived

NSC neural stem cell

CSF cerebral spinal fluid

DS Diethyl succinate

GPR91 G-protein coupled receptor 91

Sirt5 Sirtuin 5

SVZ subventricular zone

\section{Declarations}

\section{Acknowledgements}

Not applicable

\section{Author contributions}

Huang LY, Qi SH designed and coordinated the study; Ma JY, Song JX performed the experiments, acquired and analyzed data; Xu JJ, Fan HD, Cai H, Wang W, Wang YL, Hu ZL and Shen JG interpreted the data; Huang LY wrote the manuscript; all authors approved the final version of the article.

\section{Funding}

This work was supported by Grants of the National Natural Science Foundation of China (No. 81671164, 81171075), Natural Science Foundation of the Jiangsu Higher Education Institutions of China (No. 13KJA310005), and Postgraduate Research \& Practice Innovation Program of Jiangsu Province (No. KYCX19_2211, KYCX20_2502).

\section{Availability of data and materials}

The original data are available from the corresponding author on request.

\section{Ethics approval and consent to participate}

All animal experiments have been conducted after the approval of animal care committees of Xuzhou Medical University (License ID: SYXK(Su)2015-0030) and in accordance with the guidelines of the animal facility of Xuzhou Medical University.

\section{Consent for publication}

Not applicable. 


\section{Competing interests}

The authors declare that they have no competing interests.

\section{References}

Aguiar, C. J., Rocha-Franco, J. A., Sousa, P. A., Santos, A. K., Ladeira, M., Rocha-Resende, C., et al. (2014). Succinate causes pathological cardiomyocyte hypertrophy through GPR91 activation. Cell Commun. Signal. 12, 78. doi:10.1186/s12964-014-0078-2.

Beach, T. E., Prag, H. A., Pala, L., Logan, A., Huang, M. M., Gruszczyk, A. V, et al. (2020). Targeting succinate dehydrogenase with malonate ester prodrugs decreases renal ischemia reperfusion injury. Redox Biol. 36, 101640. doi:10.1016/j.redox.2020.101640.

Chen, H., Guan, B., Wang, B., Pu, H., Bai, X., Chen, X., et al. (2019). Glycyrrhizin Prevents Hemorrhagic Transformation and Improves Neurological Outcome in Ischemic Stroke with Delayed Thrombolysis Through Targeting Peroxynitrite-Mediated HMGB1 Signaling. Transl. Stroke Res. doi:10.1007/s12975019-00772-1.

Chen, H., Xu, H., Potash, S., Starkov, A., Belousov, V. V, Bilan, D. S., et al. (2017). Mild metabolic perturbations alter succinylation of mitochondrial proteins. J. Neurosci. Res. 95, 2244-2252. doi:10.1002/jnr.24103.

Chouchani, E. T., Pell, V. R., Gaude, E., Aksentijevic, D., Sundier, S. Y., Robb, E. L., et al. (2014). Ischaemic accumulation of succinate controls reperfusion injury through mitochondrial ROS. Nature 515, 431-435. doi:10.1038/nature13909.

Donega, V., and Raineteau, O. (2017). Postnatal Neural Stem Cells: Probing Their Competence for Cortical Repair. Neurosci. a Rev. J. bringing Neurobiol. Neurol. psychiatry 23, 605-615.

doi:10.1177/1073858417697036.

Eltzschig, H. K., and Eckle, T. (2011). Ischemia and reperfusion--from mechanism to translation. Nat. Med. 17, 1391-1401. doi:10.1038/nm.2507.

Etienne-Manneville, S., and Hall, A. (2003). Cdc42 regulates GSK-3beta and adenomatous polyposis coli to control cell polarity. Nature 421, 753-756. doi:10.1038/nature01423.

Gibson, G. E., Xu, H., Chen, H.-L., Chen, W., Denton, T. T., and Zhang, S. (2015). Alpha-ketoglutarate dehydrogenase complex-dependent succinylation of proteins in neurons and neuronal cell lines. $J$. Neurochem. 134, 86-96. doi:10.1111/jnc.13096.

Grimolizzi, F., and Arranz, L. (2018). Multiple faces of succinate beyond metabolism in blood. Haematologica 103, 1586-1592. doi:10.3324/haematol.2018.196097. 
Gu, W., Brännström, T., and Wester, P. (2000). Cortical neurogenesis in adult rats after reversible photothrombotic stroke. J. Cereb. blood flow Metab. Off. J. Int. Soc. Cereb. Blood Flow Metab. 20, 1166-1173. doi:10.1097/00004647-200008000-00002.

Guo, Y., Xie, C., Li, X., Yang, J., Yu, T., Zhang, R., et al. (2017). Succinate and its G-protein-coupled receptor stimulates osteoclastogenesis. Nat. Commun. 8, 15621. doi:10.1038/ncomms15621.

Hu, Z., Li, F., Zhou, X., Zhang, F., Huang, L., Gu, B., et al. (2020). Momordica charantia polysaccharides modulate the differentiation of neural stem cells via SIRT1/B-catenin axis in cerebral ischemia/reperfusion. Stem Cell Res. Ther. 11, 485. doi:10.1186/s13287-020-02000-2.

Huang, L.-Y., He, Q., Liang, S.-J., Su, Y.-X., Xiong, L.-X., Wu, Q.-Q., et al. (2014). ClC-3 chloride channel/antiporter defect contributes to inflammatory bowel disease in humans and mice. Gut 63, 15871595. doi:10.1136/gutjnl-2013-305168.

Jiang, M., Ni, J., Cao, Y., Xing, X., Wu, Q., and Fan, G. (2019). Astragaloside IV Attenuates Myocardial Ischemia-Reperfusion Injury from Oxidative Stress by Regulating Succinate, Lysophospholipid Metabolism, and ROS Scavenging System. Oxid. Med. Cell. Longev. 2019, 9137654. doi:10.1155/2019/9137654.

Kamarauskaite, J., Baniene, R., Trumbeckas, D., Strazdauskas, A., and Trumbeckaite, S. (2020). Increased Succinate Accumulation Induces ROS Generation in In Vivo Ischemia/Reperfusion-Affected Rat Kidney Mitochondria. Biomed Res. Int. 2020, 8855585. doi:10.1155/2020/8855585.

Kelly, B., and O'Neill, L. A. J. (2015). Metabolic reprogramming in macrophages and dendritic cells in innate immunity. Cell Res. 25, 771-784. doi:10.1038/cr.2015.68.

Kohlhauer, M., Dawkins, S., Costa, A. S. H., Lee, R., Young, T., Pell, V. R., et al. (2018). Metabolomic Profiling in Acute ST-Segment-Elevation Myocardial Infarction Identifies Succinate as an Early Marker of Human Ischemia-Reperfusion Injury. J. Am. Heart Assoc. 7. doi:10.1161/JAHA.117.007546.

Koronowski, K. B., Khoury, N., Morris-Blanco, K. C., Stradecki-Cohan, H. M., Garrett, T. J., and Perez-Pinzon, M. A. (2018). Metabolomics Based Identification of SIRT5 and Protein Kinase C Epsilon Regulated Pathways in Brain. Front. Neurosci. 12, 32. doi:10.3389/fnins.2018.00032.

Lampropoulou, V., Sergushichev, A., Bambouskova, M., Nair, S., Vincent, E. E., Loginicheva, E., et al. (2016). Itaconate Links Inhibition of Succinate Dehydrogenase with Macrophage Metabolic Remodeling and Regulation of Inflammation. Cell Metab. 24, 158-166. doi:10.1016/j.cmet.2016.06.004.

Li, F., He, X., Ye, D., Lin, Y., Yu, H., Yao, C., et al. (2015). NADP(+)-IDH Mutations Promote Hypersuccinylation that Impairs Mitochondria Respiration and Induces Apoptosis Resistance. Mol. Cell 60, 661-675. doi:10.1016/j.molcel.2015.10.017. 
Li, J., Yang, Y.-L., Li, L.-Z., Zhang, L., Liu, Q., Liu, K., et al. (2017). Succinate accumulation impairs cardiac pyruvate dehydrogenase activity through GRP91-dependent and independent signaling pathways:

Therapeutic effects of ginsenoside Rb1. Biochim. Biophys. Acta - Mol. Basis Dis. 1863, 2835-2847. doi:https://doi.org/10.1016/j.bbadis.2017.07.017.

Nobes, C. D., and Hall, A. (1995). Rho, rac, and cdc42 GTPases regulate the assembly of multimolecular focal complexes associated with actin stress fibers, lamellipodia, and filopodia. Cell 81, 53-62. doi:10.1016/0092-8674(95)90370-4.

Park, J., Chen, Y., Tishkoff, D. X., Peng, C., Tan, M., Dai, L., et al. (2013). SIRT5-mediated lysine desuccinylation impacts diverse metabolic pathways. Mol. Cell 50, 919-930. doi:10.1016/j.molcel.2013.06.001.

Ren, S., Yang, M., Yue, Y., Ge, F., Li, Y., Guo, X., et al. (2018). Lysine Succinylation Contributes to Aflatoxin Production and Pathogenicity in Aspergillus flavus. Mol. Cell. Proteomics 17, 457-471. doi:10.1074/mcp.RA117.000393.

Rubic, T., Lametschwandtner, G., Jost, S., Hinteregger, S., Kund, J., Carballido-Perrig, N., et al. (2008). Triggering the succinate receptor GPR91 on dendritic cells enhances immunity. Nat. Immunol. 9, 12611269. doi:10.1038/ni.1657.

Sadhukhan, S., Liu, X., Ryu, D., Nelson, O. D., Stupinski, J. A., Li, Z., et al. (2016). Metabolomics-assisted proteomics identifies succinylation and SIRT 5 as important regulators of cardiac function. Proc. Natl. Acad. Sci. U. S. A. 113, 4320-4325. doi:10.1073/pnas.1519858113.

Tannahill, G. M., Curtis, A. M., Adamik, J., Palsson-McDermott, E. M., McGettrick, A. F., Goel, G., et al. (2013). Succinate is an inflammatory signal that induces IL-1 beta through HIF-1alpha. Nature 496, 238242. doi:10.1038/nature11986.

Wang, T., Xu, Y.-Q., Yuan, Y.-X., Xu, P.-W., Zhang, C., Li, F., et al. (2019). Succinate induces skeletal muscle fiber remodeling via SUNCR1 signaling. EMBO Rep. 20, e47892. doi:10.15252/embr.201947892.

Willmes, D. M., and Birkenfeld, A. L. (2013). The Role of INDY in Metabolic Regulation. Comput. Struct. Biotechnol. J. 6, e201303020. doi:10.5936/csbj.201303020.

Xie, Z., Dai, J., Dai, L., Tan, M., Cheng, Z., Wu, Y., et al. (2012). Lysine succinylation and lysine malonylation in histones. Mol. Cell. Proteomics 11, 100-107. doi:10.1074/mcp.M111.015875.

Yang, Y., and Gibson, G. E. (2019). Succinylation Links Metabolism to Protein Functions. Neurochem. Res. 44, 2346-2359. doi:10.1007/s11064-019-02780-x.

Yang, Y., Zhang, K., Chen, X., Wang, J., Lei, X., Zhong, J., et al. (2019). SVCT2 Promotes Neural Stem/Progenitor Cells Migration Through Activating CDC42 After Ischemic Stroke. Front. Cell. Neurosci. 13, 429. doi:10.3389/fncel.2019.00429. 
Zhang, Y., Zhang, M., Zhu, W., Yu, J., Wang, Q., Zhang, J., et al. (2020). Succinate accumulation induces mitochondrial reactive oxygen species generation and promotes status epilepticus in the kainic acid rat model. Redox Biol. 28, 101365. doi:10.1016/j.redox.2019.101365.

Zhang, Z., Tan, M., Xie, Z., Dai, L., Chen, Y., and Zhao, Y. (2011). Identification of lysine succinylation as a new post-translational modification. Nat. Chem. Biol. 7, 58-63. doi:10.1038/nchembio.495.

\section{Figures}
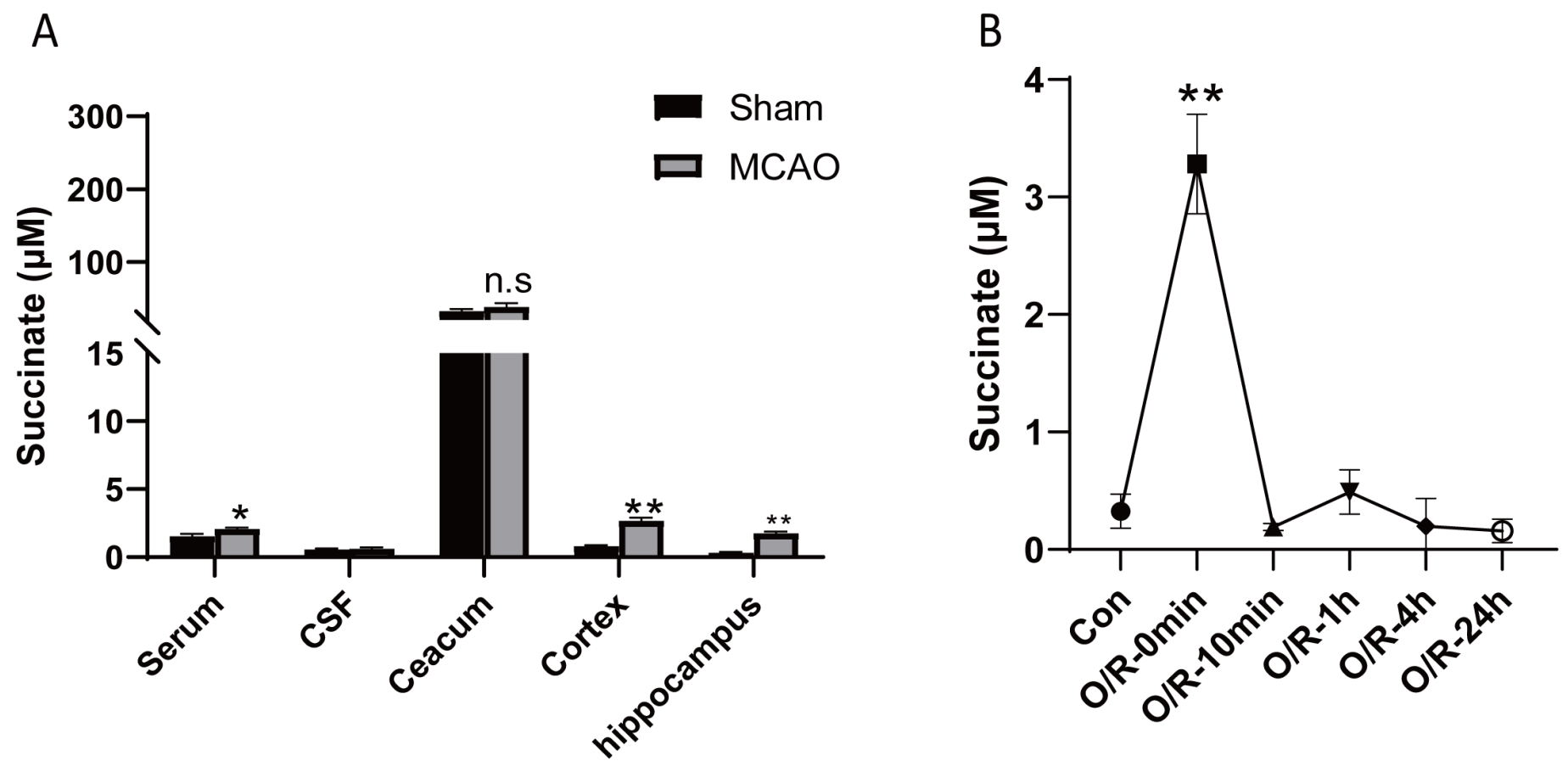

Figure 1

Succinate accumulated after ischemic/reperfusion in vivo and in vitro. A: Succinate accumulates in serum, cortex and hippocampus of MCAO rats. ${ }^{*} P<0.05$, ${ }^{*} P<0.01$, vs Sham, $n=6$. B: The level of intracellular succinate in NSCs challenged by OGD and reperfusion (O/R) for $0,10 \mathrm{~min}, 1 \mathrm{~h}, 4 \mathrm{~h}$ and $24 \mathrm{~h}$, **P $<0.01$, vs Con, $n=3$. 
A

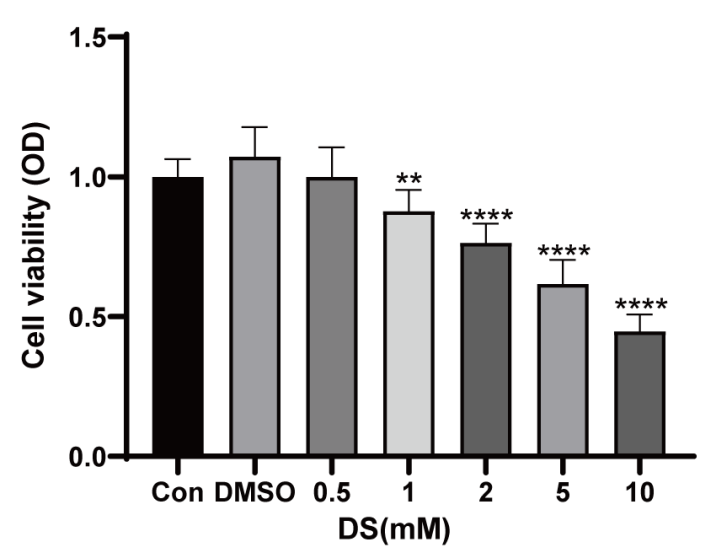

B

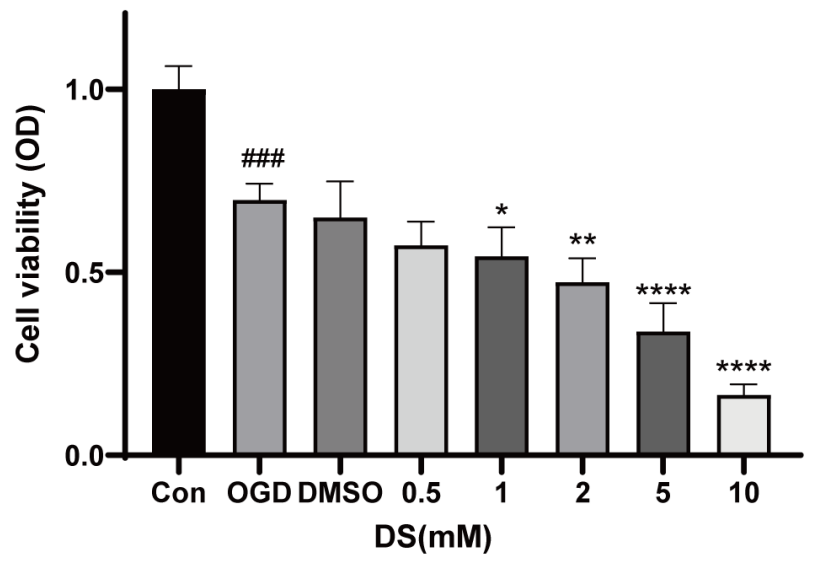

Figure 2

Diethyl succinate inhibited NSC proliferation. A: The cell viability of C17.2 NSCs treated by DS $(0.5,1,2,5$, $10 \mathrm{mM}$ ) under physiological condition. ${ }^{*} \mathrm{P}<0.01$, ${ }^{\star * \star *} \mathrm{P}<0.0001$ vs $\mathrm{DMSO}, \mathrm{n}=3$. B: The cell viability of C17.2 NSCs treated by DS $(0.5,1,2,5,10 \mathrm{mM})$ under OGD condition. ${ }^{*} P<0.05,{ }^{\star} P P<0.01,{ }^{\star} * \star * P<0.0001$ vs DMSO, \#\#\#P<0.001 vs Con, $n=3$. 
A

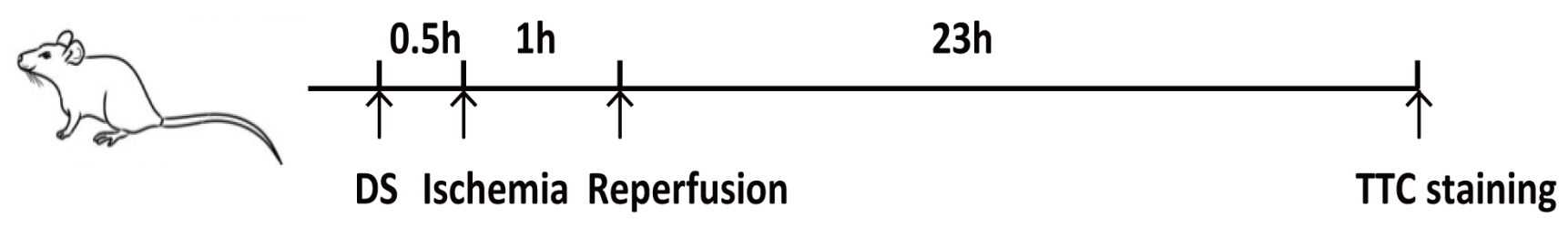

B
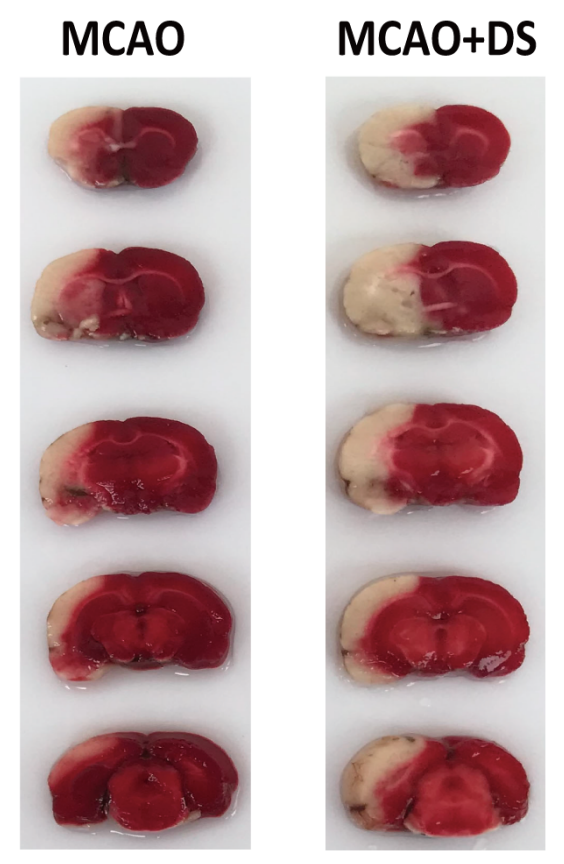

C

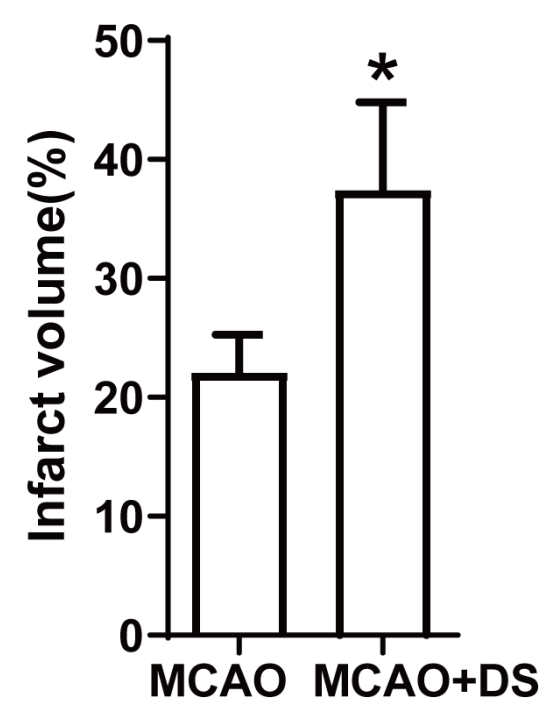

Figure 3

The level of succinate aggravated cerebral ischemic/reperfusion injury severity. A: The experimental diagram of rat MCAO model administrated with DS. B: TTC staining shows that intraperitoneally injection of DS increases infarct volume after cerebral ischemic/reperfusion injury. C: The statistical analysis of infarct volume between MCAO and MCAO plus DS group. ${ }^{*} \mathrm{P}<0.05$, vs Con; $\mathrm{n}=6$. 
A
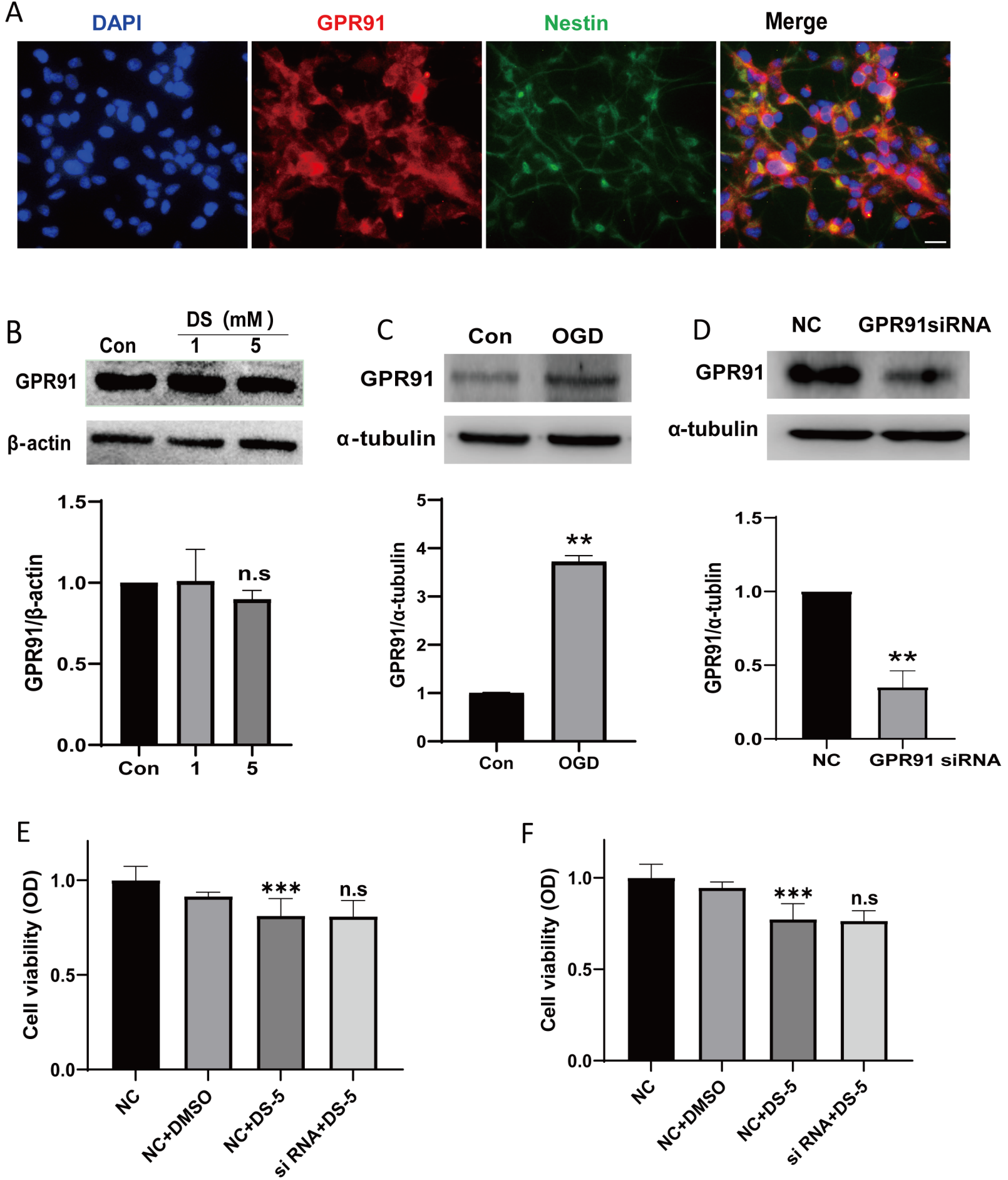

Figure 4

Succinate receptor GPR91 was dispensable for DS inhibiting NSC proliferation after OGD challenge. A:Succinate receptor GPR91 is expressed on primary neural stem cells. Scale bar, $50 \mu \mathrm{m}$. B:The expression of GPR91 in C17.2 challenged by $1 \mathrm{mM}$ and $5 \mathrm{mM}$ DS under physiological condition. n.s indicated P>0.05 vs Con. C: The expression and statistical analysis of GPR91 in C17.2 cell challenged by OGD. ${ }^{*} \mathrm{P}<0.01$, vs Con; $\mathrm{n}=3$. D: The statistical analysis of the expression of GPR91 in C17.2 NSCs treated 
by OGD. ${ }^{*} \mathrm{P}<0.01$, vs Negative control (NC); $n=3$. E: The effect of GRP91 knockdown on DS induced proliferation assessed by CCK8. ${ }^{* \star *} P<0.001$, vs NC+DMSO; n.s indicated $P>0.05$ vs NC+DS-5, $n=3$. F: The effect of GRP91 knockdown on DS induced proliferation under OGD condition assessed by CCK8. $\star \star * P<0.001$, vs NC+DMSO; $n$.s indicated $P>0.05$ vs $N C+D S-5, n=3$.
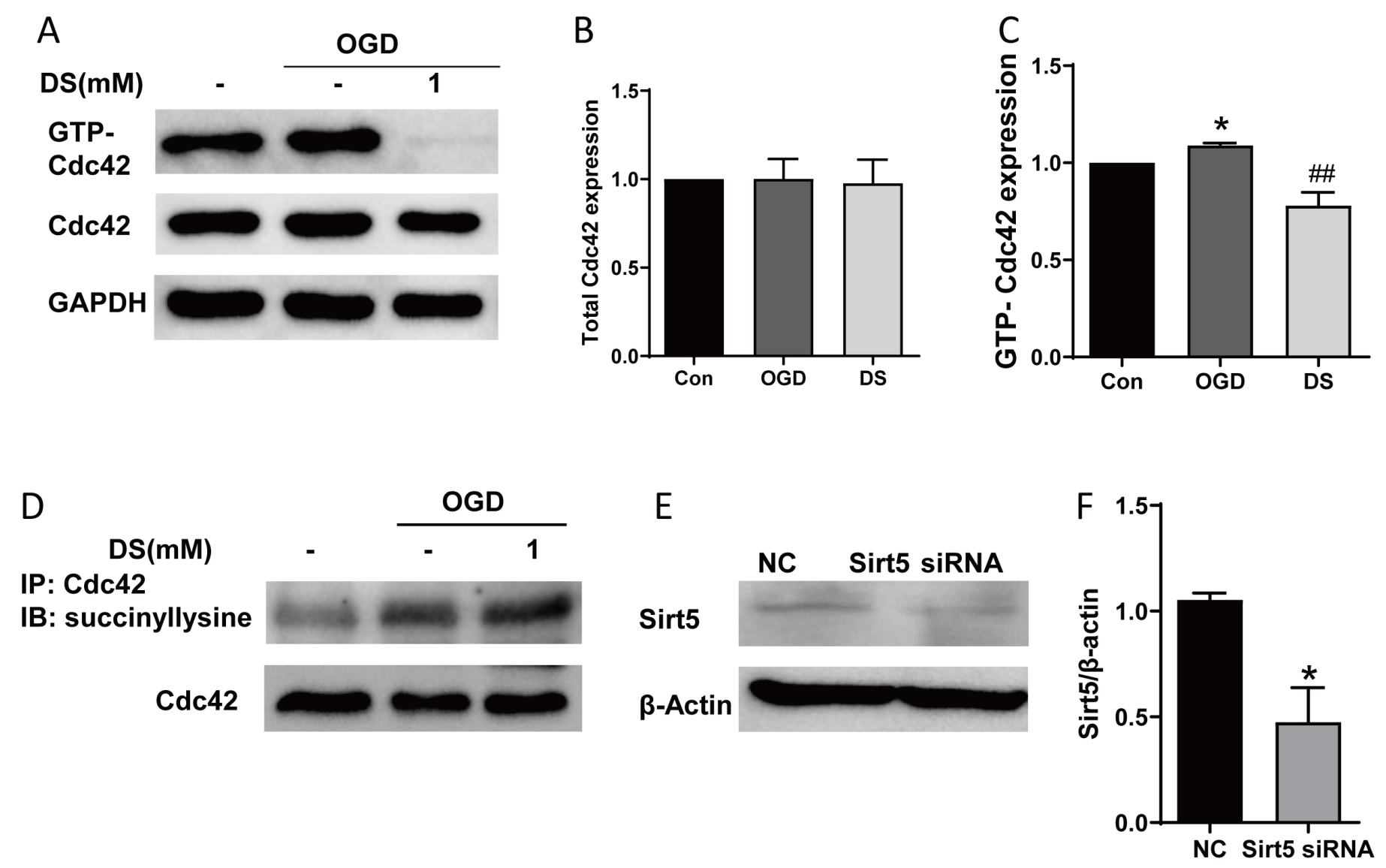

G

NC Sirt5 siRNA

IP: Cdc42

IB: succinyllysine

GTP-Cdc42

Cdc42
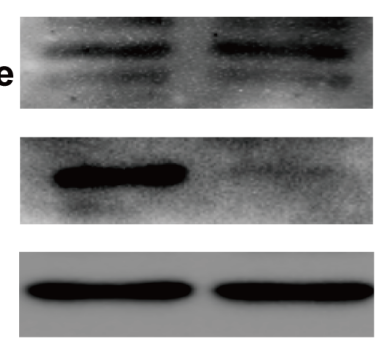

Figure 5

DS inhibited GTP-Cdc42 activity by increasing Cdc42 succinylation. A: Cdc42 GTPase activity was performed using the Rac1/Cdc42 activation assay. B,C: The statistical analysis of the level of total Cdc42 and GTP-Cdc42 in C17.2 NSCs treated by DS. *P<0.05, vs Con; \#\#P<0.01, vs OGD; $n=3$. D: The Cdc42 
succinylation was assayed by Co-IP. E: The effect of Sirt5 knockdown on the expression of Sirt5 in NSCs was detected by western blotting. F: The statistical analysis of the expression of Sirt5 in NSCs after Sirt5 knockdown. ${ }^{*}<<0.05$, vs Con; $n=3$. Increase of $\mathrm{Cdc} 42$ succinylation reduces GTP-Cdc42 activity. G: Increase of Cdc42 succinylation by sirt5 siRNA transfection represses the level of GTP-Cdc42.

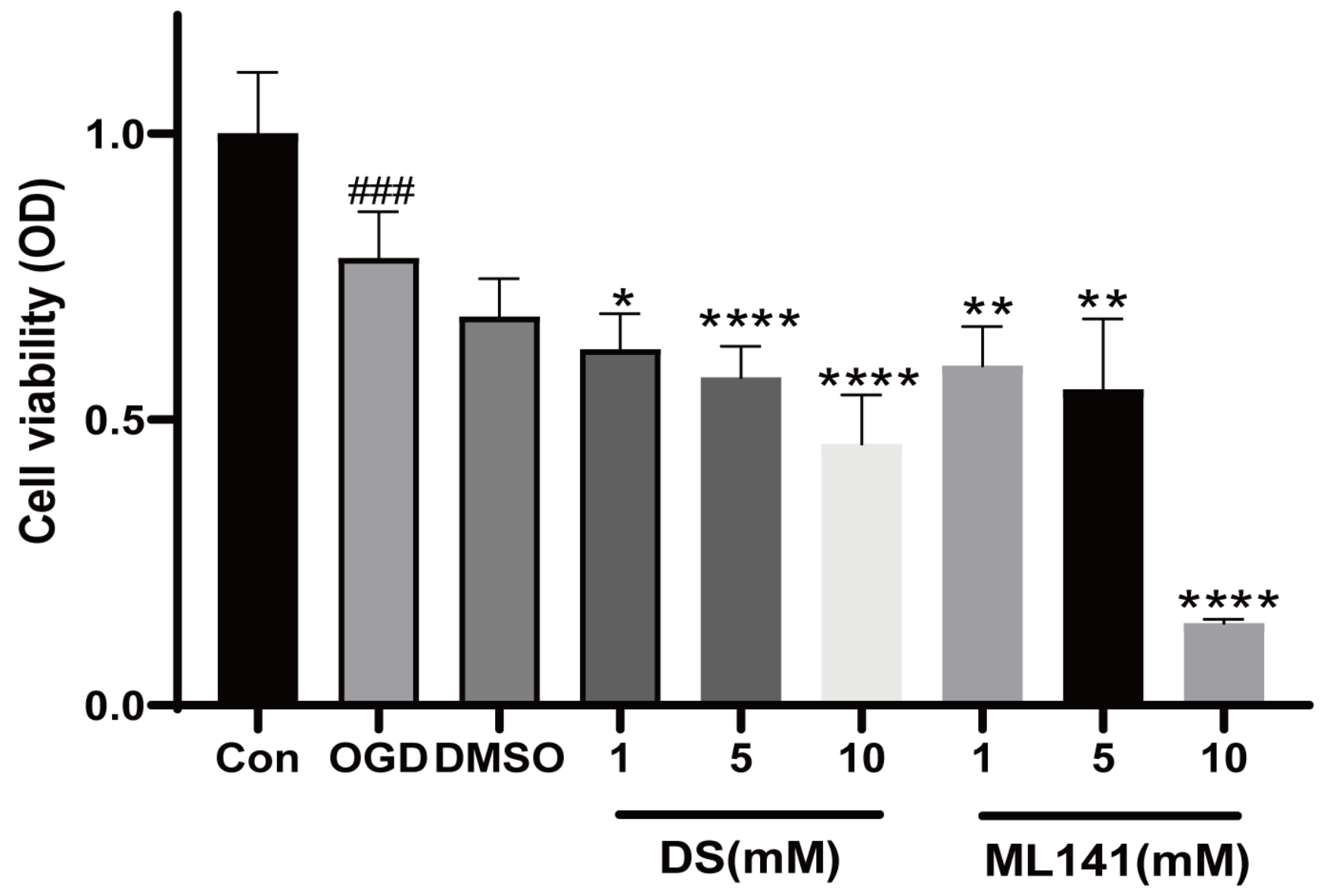

Figure 6

ML141 inhibited NSC proliferation under OGD condition. The effect of DS and ML141 with the same concentration on NSC proliferation under OGD condition was detected by CCK8 counting. ${ }^{*} \mathrm{P}<0.05$, $\star \star P<0.01, * \star \star \star P<0.0001$ vs DMSO, \#\#\#P<0.001 vs Con, $n=3$.

\section{Supplementary Files}

This is a list of supplementary files associated with this preprint. Click to download.

- Graphicabstract.tif 